Cahiers d'études italiennes

19 | 2014

FILIgRANA

Idées et formes du tragique dans la société et la culture italiennes

\title{
«Questa miseranda tragedia ». Le sac de Rome, la providence, la politique
}

"Questa miseranda tragedia». Il sacco di Roma, la provvidenza, la politica

Jean-Claude Zancarini

\section{(2) OpenEdition}

Journals

Édition électronique

URL : http://journals.openedition.org/cei/2142

DOI : $10.4000 /$ cei.2142

ISSN : 2260-779X

Éditeur

UGA Éditions/Université Grenoble Alpes

Édition imprimée

Date de publication : 1 novembre 2014

Pagination : 111-125

ISBN : 978-2-84310-285-1

ISSN : 1770-9571

Référence électronique

Jean-Claude Zancarini, « «Questa miseranda tragedia ». Le sac de Rome, la providence, la politique », Cahiers d'études italiennes [En ligne], 19 | 2014, mis en ligne le 01 mai 2016, consulté le 26 mars 2021. URL : http://journals.openedition.org/cei/2142 ; DOI : https://doi.org/10.4000/cei.2142 


\title{
«QUESTA MISERANDA TRAGEDIA ». LE SAC DE ROME, LA PROVIDENCE, LA POLITIQUE
}

\author{
Jean-Claude Zancarini \\ ENS de Lyon
}

La période des guerres d'Italie du XVI ${ }^{\mathrm{e}}$ siècle a souvent été désignée comme une tragédie. Pour ne prendre que quelques exemples, Raffaello Ramat publia en 1953 chez Olschki un livre intitulé Il Guicciardini e la tragedia d'Italia et, en I995, l'un des contributeurs d'un livre collectif important sur le début de ces guerres ${ }^{1}$, intitule sa contribution sur les rapports entre Ferrante d'Aragon et Galeazzo Maria Sforza "Towards the Tragedia d'Italia ${ }^{2}$ ». Il est vrai que la métaphore vient facilement à l'esprit. Une situation de départ que les contemporains décrivent comme un âge d'or, le surgissement d'ennemis d'outre-monts, les Français du roi Charles VIII, qui en I494 viennent bouleverser cette situation bienheureuse, les actes successifs qui voient s'introduire sur la scène de l'histoire d'autres protagonistes (les Espagnols, les Allemands, les Suisses), les renversements successifs et brutaux des rapports de force entre les belligérants et, enfin, la catastrophe finale, le sac de Rome de mai 1527 , qui entraîne la soumission de l'Italie aux Espagnols, pendant de longues décennies. Pourtant, le terme tragedia n'est que très rarement utilisé par les contemporains des guerres pour désigner ce qu'ils voient se dérouler sous leurs yeux: ils utilisent plutôt d'autres expressions comme le calamità d'Italia ou la ruina d'Italia. Un indice textuel, dans une lettre de Machiavel à Francesco Guicciardini, me paraît toutefois à souligner car il indique que ce sentiment d'être dans une tragédie n'était pas absent. C'est une lettre datée post 2I octobre 1525, signée "Niccolò Machiavelli istorico, comico e tragico"; c'est la seule utilisation

I. D. Abulafia (éd.), The French Descent into Renaissance Italy I494-95: Antecedents and Effects, Aldershot (G.B.), Variorum, 1995.

2. V. Ilardi, «Towards the Tragedia d'Italia: Ferrante and Galeazzo Maria Sforza, Friendly Enemies and Hostile Allies», The French Descent..., ouvr. cité, p. 9I-I22. 
du terme tragico dans l'ensemble des œuvres et des lettres du Secrétaire florentin. On comprend aussitôt pourquoi cette triple définition si on se réfère aux trois paragraphes qui précèdent la signature :

Il Morone ne andò preso, et il ducato di Milano è spacciato; et come costui ha aspettato il cappello, tutti gli altri principi l'aspetteranno, né ci è più rimedio. Sic datum desuper.

Veggio 'n Alagna tornar lo fiordaliso

e nel vicario suo, etc.

Nosti versus, cetera per te ipsum lege. Facciamo una volta un lieto carnesciale, et ordinate alla Barbera uno alloggiamento tra quelli frati, che, se non inpazzano, io non ne voglio danaio, et raccomandatemi alla Maliscotta, et avvisate a che porto è la commedia, et quando disegnate farla.

Io hebbi quello augmento insino in cento ducati per la Istoria. Comincio ora a scrivere di nuovo, et mi sfogo accusando i principi, che hanno fatto tutti ogni cosa per condurci qui. Valete 3 .

Sans faire un commentaire détaillé, on peut souligner que chacun des titres que se donne Machiavel renvoie à un des paragraphes précédents : istorico parce qu'il est en train d'écrire les Istorie fiorentine et que son salaire vient d'être augmenté; comico parce qu'à cette époque est prévue une représentation de La Mandragola à Faenza. Il est donc tragico parce qu'il s'intéresse aux événements qui se déroulent de son temps et que ces événements sont tragiques: Morone, chancelier du duc de Milan Francesco II Sforza, vient de se faire "encapuchonner" (ha aspettato il cappello est une expression du langage de la fauconnerie) par le marquis de Pescara, chef des troupes espagnoles dans le duchét, et le même sort attend les autres princes d'Italie; quant au pape, c'est avec deux vers de Dante's sur Boniface VIII que Machiavel annonce ses déboires à venir, rejoignant ainsi, de façon étonnante, la prédiction plus ancienne de Fra Girolamo Savonarola : "Roma ha 'andare sotto sopra! ${ }^{16}$ " Les deux prophètes de malheur allaient en effet voir leur prophétie se réaliser en mai I 527 avec le sac de Rome, miseranda tragedia. Avant de voir comment Luigi et Francesco Guicciardini relatent l'événement inouï, la catastrophe, que fut le sac de Rome en mai I527, voyons comment, plus globalement, les Florentins donnent sens aux «signes extraordinaires» et aux «grands événements».

3. N. Machiavelli, Opere, éd. de C. Vivanti, Turin, Einaudi, I999, vol. II, p. 4II.

4. Girolamo Morone fut emprisonné le I5 octobre I525. Francesco II Sforza se réfugia dans le château de Milan.

5. Dante, Purgatorio, XX, v. 86-87.

6. Un exemple dans les Prediche sopra i Salmi, p. 42 : "l'Italia ha tutta 'andare sotto sopra, e Roma, e dipoi si ha a rinovare la Chiesa..." 


\section{Les «signes » et les « effets » : lecture providentialiste et lecture politique}

En effet les écritures politiques des auteurs-acteurs politiques de Florence (on pense ici au premier chef à Machiavel et à Francesco Guicciardini) partent d'un questionnement lié à la prégnance des événements : comment agir et écrire en tenant compte "des temps», de la "qualité des temps»? Un des aspects de cette réflexion sur la nouveauté des événements porte sur les "grands événements" et les «signes extraordinaires": on trouve des indices qui indiquent que l'on est en train de passer d'une conception "providentielle» des événements voulus par «la fortune» ou par Dieu (on se souviendra que Commynes, au moment de relater «l'emprise [...] tres deraisonnable» de Charles VIII, commente : "Ainsi fault conclure que ce voyage fut conduict de Dieu tant a l'aller que au tourner ${ }^{7}$ ») à une analyse qui met l'accent sur leur sens essentiellement historico-politique.

Pour les contemporains, l'arrivée en Italie du roi de France Charles VIII en 1494 fut annoncée par des "signes célestes» qui indiquaient que de grands événements allaient se dérouler et Machiavel et Guicciardini ne sont pas les derniers à rapporter les nombreux «segni celesti, predizioni, pronostichi e prodigi». Par exemple, celui-ci, relaté tant par Machiavel (Discours, I, LVI) que par Guicciardini (Storia d'Italia, I, IX) : dans le ciel, près d'Arezzo, on a vu des hommes armés, montés sur des chevaux gigantesques, au milieu d'un tintamarre de trompettes et de tambours; certains, dont Machiavel, précisent que ces hommes combattaient entre eux. Il n'y a aucun doute pour Guicciardini comme pour Machiavel : ces signes ont vraiment existé. Guicciardini rapporte que ça se voyait nettement, visibilmente, Machiavel que "chacun le sait» (sa ciascuno...). D'ailleurs le chapitre dans lequel Machiavel relate ce "prodige ou ce signe céleste» s'intitule "Innanzi che seguino i grandi accidenti in una città o in una provincia, vengono segni che gli pronosticono, o uomini che gli predicano". Il émet une hypothèse explicative ("potrebbe essere che, sendo questo aere, come vuole alcuno filosofo, pieno di intelligenze, le quali per naturali virtì preveggendo le cose future, ed avendo compassione agli uomini, acciò si possino preparare alle difese, gli avvertiscono con simili segni»). Et il conclut en disant que "comunque e' si sia, si vede cosi essere la verità; e che sempre dopo tali accidenti sopravvengono cose istraordinarie e nuove alle provincie»" ${ }^{8}$.

7. Philippe de Commynes, Mémoires, VII, 2, éd. Blanchard, p. 490.

8. Dans les notes de son édition des Discorsi, Corrado Vivanti estime que l'écho de la culture néoplatonicienne de la Florence de Marsile Ficin et de Pic de la Mirandole est évident dans ce chapitre (N. Machiavelli, Opere, éd. de C. Vivanti, Turin-Paris, Einaudi-Gallimard, 1997, vol. I, p. 993). 
Comment faut-il interpréter ces textes? D’abord, et ça n'a évidemment rien de surprenant si l'on a une démarche d'approche des textes fondée sur l'histoire (mais ça pourrait l'être pour ceux qui voient en Machiavel le théoricien de la politique moderne ne craignant ni Dieu ni diable!), il faut tout simplement admettre que Guicciardini et Machiavel ont les croyances et superstitions de leur époque (Guicciardini croit aussi que l'air est plein d'esprits : il écrit même dans un de ses ricordi qu'il en a eu la preuve manifeste $^{9}$ ). Mais les signes célestes - ces prodiges qui paraissent inexplicables, surnaturels — sont à interpréter et c'est là que les «effets" politiques et militaires deviennent déterminants. La formulation de Guicciardini, dans la Storia d'Italia, est la plus nette, mais on peut arriver à la même conclusion pour Machiavel. Voici ce qu'écrit Guicciardini, après avoir fait la liste des signes célestes qui ont annoncé «les calamités futures»: "Ma a" segni celesti, predizioni, pronostichi e prodigi accresceva ogni di piú la fede l'appropinquarsi degli effetti». Ce sont donc les effetti (les faits et les effets) qui permettent de donner sens aux «signes célestes, prédictions, pronostics et prodiges» et pas le contraire. L'analyse par les signes célestes est, par elle-même, impossible. Il y a des signes célestes, de même qu’il y a peut-être des desseins de la Providence, mais les hommes ne peuvent les atteindre, ce sont des abyssus multa ${ }^{\mathrm{IO}}$, et du coup on ne peut prévoir les "choses futures». Les hommes ne peuvent se déterminer qu'en partant des effets, non des signes, par une analyse historique à rebours, non par une inspiration divine qui permettrait de "prédire». On retrouve la même idée chez Machiavel, dans un passage des Istorie fiorentine, où il relate la mort de Laurent de Médicis, en rappelant un des prodiges qui figuraient en Discours (I, LVI) et qui avaient annoncé la mort du Magnifique : «E come dalla sua morte ne dovesse nascere grandissime rovine ne mostrò il cielo molti evidentissimi segni»; un peu plus loin, en employant le même terme que Guiccardini - effetto - il précise que cette « ruine» qu'allait provoquer la disparition de Laurent «lo dimostrò poco di poi lo effetto».

9. F. Guicciardini, Ricordi, edizione critica a cura di Raffaele Spongano, Florence, Sansoni, I95I, C 2II, p. 223 : "Io credo potere affermare che gli spiriti siano; dico quella cosa che noi chiamiamo spiriti, cioè di quelli aerei che dimesticamente parlano con le persone, perché n'ho visto esperienzia tale che mi pare esserne certissimo; ma quello che siano e quali, credo lo sappia si poco chi si persuade saperlo quanto chi non vi ha punto di pensiero. Questo, e el predire el futuro, come si vede fare talvolta a qualcuno o per arte o per furore, sono potenzie occulte della natura, o vero di quella virtú superiore che muove tutto; palesi a lui, segreti a noi, e talmente, che e cervelli degli uomini non vi aggiungono."

Io. Voir infra le texte du ricordo C 92 d'où est tirée l'expression. 


\section{Le sac de Rome dans la Storia d'Italia de Francesco Guicciardini et le Sacco di Roma de Luigi Guicciardini}

Francesco et Luigi Guicciardini, l'un dans le livre XVIII de sa Storia d'Italia (mais également dans sa Consolatoria et ses lettres ${ }^{\mathrm{II}}$ ), l'autre dans son Sacco di Roma, ont relaté l'" accidente atroce e miserabile ${ }^{\mathrm{I2}}$, la più mesta, la più spaventevole e la più vergognosa tragedia ${ }^{13}$ que fut le sac de la ville éternelle. L'analyse de ces récits permet de mettre en évidence comment fonctionne le rapport entre «signes» et "effets», entre lecture providentialiste et lecture proprement politique d'une catastrophe.

\section{«La giusta ira di Dio ». Le sac de Rome décrit par Luigi Guicciardini ${ }^{14}$}

Dans le Sacco di Roma, écrit après l'événement puis dédié à Côme de Médicis, Luigi Guicciardini a voulu "notare parte dei miserandi casi successi in questi prossimi giorni in Roma» en insistant sur le fait que cette façon de faire n'est pas habituelle chez les historiens ("benchè non sia stato costume de' passati istorici scrivere, se non generalmente, tutti i notabili infortunii e ruine successe nell'espugnate città»); avant de décrire ces miserandi casi, Luigi présente " $i$ principali casi seguiti dal principio di questa lega insieme con la sua ruina» et l'essentiel des deux livres dont se compose le texte est consacré à l'analyse de la guerre qui suit la signature de la ligue de Cognac en mai I526, le premier livre esquissant les événements qui vont de cette signature de la ligue à avril I527; le second rendant compte avec plus de précision de ce qui se passa à Florence (où Luigi était gonfalonier de justice) puis de la prise de Rome et du sac. Un des aspects de la lecture de

II. Sur la façon dont Francesco Guicciardini relate l'événement dans ses lettres, voir Hélène Miesse, «Regards croisés sur le sac de Rome : Le "carteggio" de Francesco Guicciardini et les "Diarii" de Marino Sanudo", dans P. Moreno, L. Godinas et H. Miesse (éd.), Encuentros/Desencuentros. Italia y España en los siglos XV y XVI: Textos y contextos. Actas del congreso internacional (UNAM-Instituto de Investigaciones bibliogràficas, 26-27 octobre 2009), sous presse.

I2. F. Guicciardini, Consolatoria, dans Autodifesa di un politico, Bari-Rome, Laterza, I993, p. 92.

I3. L. Guicciardini, Il sacco di Roma, édition citée dans la note suivante, p. 7. Sur Luigi Guicciardini (I478I55I), voir la notice de M. Doni, dans le D.B.I. (Dizionario Biografico degli Italiani). Dans son livre sur le sac de Rome, André Chastel, reprenant la fausse attribution des premières éditions parisiennes, attribue cette citation à Francesco Guicciardini (A. Chastel, Le sac de Rome, I527, Paris, Gallimard, 1984, p. 134).

I4. J'utilise l'édition électronique de Danilo Romei (<www.nuovorinascimento.org $>$ ) qui reprend l'édition établie par C. Milanesi en I867 : Il sacco di Roma del MDXXVII. Narrazioni di contemporanei scelte per cura di Carlo Milanesi, Florence, G. Barbèra Editore, I867, p. I-244; l'indication des pages de l'édition originale est donnée entre crochets carrés. On ne connaît pas la date exacte de rédaction du texte. Dans le D.B.I., M. Doni, le rédacteur de la notice "Luigi Guicciardini», estime qu’il a été probablement écrit Io ans après les faits, mais ne donne pas d'arguments. Dans la préface Luigi Guicciardini laisse entendre qu'il a écrit peu après les faits mais a attendu pour le dédier au duc Côme Ir (voir infra note I6). Le Sacco fut publié à Paris en I664 puis en I758, sous le nom de Francesco Guicciardini. 
Luigi Guicciardini est incontestablement providentialiste; il estime que le sac de Rome est l'expression de la giusta ira di Dio ${ }^{15}$ et rappelle à ses lecteurs les straordinari segni et les portenti qui annoncèrent la catastrophe. Il me semble toutefois possible de montrer que, pour Luigi Guicciardini, le courroux de Dieu est très lié aux erreurs des hommes, dont beaucoup sont d'ordre politique et militaire; l'expression l'ira di Dio revient à trois reprises dans le texte dans des contextes qui mettent en évidence le lien complexe entre dessein divin et agir politique. Le premier de ces passages sert de conclusion au premier livre du Sacco di Roma:

Questi vari e gravi effetti, seguiti dal principio di tanto santa e veneranda lega, insino all'arrivo de' Cesariani in Valdarno di sopra, se fussero stati da me narrati più particularmente, e scritti con quell'ordine che interamente si richiede alle regole dell'istoria, mostrerebbono piu apertamente a qualunque, quanto la fortuna abbia sempre accompagnato gl'Imperiali e quanta tardità e timore sia stato sempre nell'esercito della lega. Nondimeno, stimando che saranno da altri, più di me diligenti, e composti e scritti, volentieri gli ho pretermessi, e lasciato a loro questa intera descrizione. Perchè l'animo mio non è stato disposto a narrare la maggior parte delle cose seguite nei giorni miei (come molt'altri istorici lodevolmente ne' loro hanno scritto), ma solamente contare questo vituperoso sacco di Roma, accio che in qualche parte apparisca, in che ruina ed esterminio pervengano quelli stati e quelli governi, che sono male consigliati, e peggio nelle loro imprese ammaestrati. Forse per l'avvenire mi sforzerò molto particularmente porre innanzi agli occhi di ciascuno che leggerà, le rapine, li strazi, i sacrilegii e le crudeltà usate continuamente in questi lagrimevoli giorni, ne' quali ora scrivo, da' perfidi Tedeschi e dalli Spagnoli, in tanto fiera e cosi nobile città; sarà perchè meglio si conosca per ciascuno in futuro la giusta ira di Dio. Per la qual cosa, coloro che le repubbliche e li principati governano e consigliano, doverebbono imparare a esser savi alle spese d'altri, perchè nelle proprie ruine difficilmente si può più d'una volta farne esperienza. (Sacco..., p. I23-I25; je souligne.)

On constate l'insistance sur les erreurs ("tardità e timore») commises par les chefs militaires de la "santa e veneranda lega", celles que commettent "quelli stati e quelli governi, che sono male consigliati, e peggio nelle loro imprese ammaestrati»; quant à la giusta ira di Dio, Luigi espère que "coloro che le repubbliche e li principati governano e consigliano" en tireront les leçons nécessaires. Sur ces points, d'autres passages du texte permettent d'expliciter les analyses de Luigi. Sur la "tardità e timore» de l'armée de la ligue, Luigi met en cause les décisions du capitaine des

15. Ce point a déjà été signalé par Rudolf von Albertini : "Il sacco della città eterna è anche per lui [= Luigi Guicciardini] la giusta ira di Dio. Il problema religioso si pone nel Guicciardini in termini analoghi a quelli del Vettori. Anche in lui un anticurialismo particolarmente violento e nel contempo [...] il riconoscimento del cristianesimo e l'idea di una riforma interna della Chiesa." (R. von Albertini, Firenze dalla repubblica al principato, Turin, Einaudi, 1995 [I $\mathrm{I}^{\mathrm{re}}$ éd. Bern, I955], p. 268.) 
troupes vénitiennes, le duc d'Urbin Francesco Maria della Rovere, sur lequel il porte des jugements aussi négatifs que ceux que portera son frère Francesco et dont il estime, après avoir écrit, non sans ironie, "mi voglio persuadere che la dolorosa sorte d'Italia facessi a quel duca pigliare sempre i più dannosi partiti» (Sacco..., p. 33), qu'il a fait volontairement tout ce qu'il pouvait faire pour se venger des Médicis ("Per questo sdegno [il duca d'Urbino] non ha mai soccorso il papa, nè soccorrerà per lo avvenire ancora; ma dove potrà offendere e nuocere crudelmente la casa dè Medici, non si straccherà mai», Sacco..., p. 22I); ce sont ces erreurs volontaires qui ont fait la fortuna des impériaux. Les erreurs de «coloro che le repubbliche e li principati governano e consigliano" ne manquent pas dans le texte : Luigi insiste sur les erreurs de jugement de Clément VII ("errore» lorsqu’il signe la trève avec le vice-roi de Naples, "errore [...] più manifesto e gravissimo" lorsque, peu après, il licencie les Suisses et les soldats des Bandes noires qui auraient pu défendre Rome; "gravissimo errore» lorsqu'il accepte que le duc d'Urbin dirige de fait l'armée de la ligue); au-delà des responsabilités et des erreurs individuelles, Luigi met en lumière, dès le proemio du texte, le rôle de "l'avaro, ambizioso ed oziosissimo governo de' moderni prelati» et dénonce, dans les premières pages du second livre, l'«incomparabile errore de' moderni» qui consiste à ne pas être "delle proprie armi armato".

Il y a dans cette mise en évidence un lien très net avec les thèses machiavéliennes, non seulement celles qui mettent en avant la nécessité des arme proprie, mais également celles qui rendent l'église responsable de cet état de fait. La question du lien entre la présence de l'Église sur le sol italien et l'abandon des armes propres est clairement mise en évidence dans le chapitre XII du Prince:

Avete dunque a intendere come, tosto che in questi ultimi tempi lo imperio cominciò a essere ributtato di Italia, e che il papa nel temporale vi prese più reputazione, si divise la Italia in più stati; perché molte delle città grosse presono l'arme contra a' loro nobili, li quali, prima favoriti dallo imperatore, le tennono oppresse; e la Chiesia le favoriva per darsi reputazione nel temporale; di molte altre e' loro cittadini ne diventorono principi. Onde che, essendo venuta l'Italia quasi che nelle mani della Chiesia e di qualche Repubblica, et essendo quelli preti e quelli altri cittadini usi a non conoscere arme, cominciorono a soldare forestieri. (Chap. XII, 28-29; je souligne.)

Par ailleurs, le chapitre I xxx des Istorie fiorentine est consacré à l'analyse du processus historique qui détermine la place prépondérante de l'Église en Italie. C'est ce processus historique qui, selon Machiavel, conduit au mondo guasto (Istorie fiorentine, $\mathrm{V}, \mathrm{I}$ ), au monde privé de vertu de questi nostri corrotti tempi (Discorsi, II, XIX). On remarquera que Luigi Guicciardini, outre la proximité des thèses qu'il avance, emploie lui-même 
des expressions très proches de celles de Machiavel : «in un tempo tanto corrotto, ed in una milizia tanto disordinata e timida" (Sacco..., p. 76). On se rend bien compte que, dès lors, l'ira di Dio est, pour Luigi, liée à une série d'erreurs commises par les capi et il précise que selon lui les responsables de cet état de fait sont avant tout les capi ecclesiastici ("mercè de' capi, non tanto secolari, quanto ecclesiastici, che l'hanno [= l'Italia] continuamente guidata e comandata", Sacco..., p. I32).

Le second passage où il est fait mention de la colère divine est particulièrement net de ce point de vue et permet de comprendre plus précisément quel est le sens des erreurs commises :

Laonde l'esempio de' nostri antenati molto ci vitupera, considerato che, quando quattro, quando sei, e quando I2,000 oltramontani poco esperti, male armati, e privi di proprio capitano travaglino, consumino e sottomettino questa nostra provincia, talmente, che $i$ savi sabbandonino e con gl'ignoranti affermino dicendo, non restare a noi rimedio alcuno, per procedere tanto flagello dall'ira di Dio, e che per $i$ nostri gravissimi errori meritiamo tanto male e peggio: querele e rammarichi (secondo il giudizio mio) di uomini troppo abbandonati, interamente privi di quella generosità, che è naturale all'uomo. Perchè qual'esperienza o qual religione c'impedisce che, se noi vogliamo, noi possiamo con li buon costumi e colle virtuose opere, mutare, quando verso di noi irata sia (cristianamente parlando) la divina sentenza? perchè senza dubbio la somma bontà si piegherebbe facilmente ogni volta vedesse negli animi nostri l'amore della povertà e della giustizia, ed esser pronti e disposti volere con le proprie e ordinarie armi ostinatamente difendere e la vita e la patria, o valorosamente morire. Ma se, per le persuasioni e promissioni di qualche moderno profeta, stimiamo, nella copia di tanti effeminati e abbominandi vizi, e in tanta pigrizia e pusillanimità costituiti, esser da Dio massimo aiutati e liberati; certamente con troppa ignoranza erriamo, e invano aspettiamo il celeste aiuto: perchè, se non muteremo modo, non muterà sentenza, ma più l'un giorno che l'altro, si mostrerà sopra le teste nostre gravissima. (Sacco..., p. I29I30; je souligne.)

Une partie de la réflexion de Luigi ressemble beaucoup à celle que l'on trouve dans le chapitre XXIV du Prince lorsque Machiavel énonçait que les princes qui ont perdu leur état devaient accuser, non la fortune, mais leur paresse et leur lâcheté (XXIV, 8 : "non accusino la fortuna, ma la ignavia loro»); ceux qui estiment qu'il s'agit d'un fléau voulu par Dieu à cause des très graves erreurs des hommes et qu'il n'y a donc pas de remède à la situation, sont «interamente privi di quella generosità, che ̀̀ naturale all'uomo». On comprend que les très graves erreurs des hommes concernent les choix politiques et militaires (il aurait fallu «esser pronti e disposti volere con le proprie e ordinarie armi ostinatamente difendere e la vita e la patria»), mais aussi une façon erronée de concevoir la religion en abandonnant "l'amore della povertà e della giustizia». Luigi semble commenter le passage du Prince dans lequel Machiavel, sans le citer, fait 
allusion à Savonarole (XII, 9 : «chi diceva come n'erono cagione e peccati nostri, diceva il vero; ma non erano già quegli che credeva, ma questi che io ho narrati... »); pour Machiavel «les péchés des princes» consistaient à avoir fait confiance aux armes mercenaires (XII, 8 : «la ruina di Italia non è causata da altro che per essersi per spazio di molti anni riposata tutta in sulle armi mercennarie»). Luigi Guicciardini, dans le passage que nous avons cité, estime donc que les erreurs humaines sont à la fois d'ordre politicomilitaire et religieux. Il n'en reste pas moins que si ces erreurs politiques et militaires n'avaient pas été faites, le fléau divin ne serait sans doute pas venu car, Luigi le dit clairement, la religion n'interdit pas de faire preuve de courage et de se battre avec ses "armes propres» ("esser pronti e disposti volere con le proprie e ordinarie armi ostinatamente difendere e la vita e la patria, o valorosamente morire»). On entend là encore une fois un écho de thèses machiavelliennes, celle sur la nécessité des "armi proprie», bien sûr, mais aussi celle qui est exprimée dans les Discours (II, II) lorsque Machiavel critique ceux qui "ont interprété notre religion selon l'oisiveté et non selon la vertu»:

E benché paia che si sia effeminato il mondo, e disarmato il Cielo, nasce più sanza dubbio dalla viltà degli uomini, che hanno interpretato la nostra religione secondo l'ozio, e non secondo la virtù. Perché, se considerassono come la ci permette la esaltazione e la difesa della patria, vedrebbono come la vuole che noi l'amiamo ed onoriamo, e prepariamoci a essere tali che noi la possiamo difendere.

Il y a donc, chez Luigi Guicciardini, une double explication de l'événement; l'une tend à en tirer les leçons politiques et militaires; l'autre à mettre en lumière les raisons religieuses de la colère divine qui s'est, il n'en doute pas, exprimée avec force ${ }^{\mathrm{I}}$. Cette double explication n'exonère en rien les hommes de leur responsabilité. Le dernier passage où Luigi fait allusion à la juste colère divine met en scène les straordinari segni qui ont annoncé le sac de la ville éternelle :

Potrei narrare ancora qualche portento accaduto non molto tempo innanzi in Roma, significante la ruina grande e propinqua: come il partorire di una mula nel palazzo della Cancelleria, e spontaneamente pochi giorni avanti esser ruinata una gran parte di quelle mura, che congiugnevano il palazzo con il castello, se non mi ritenessi il conoscere, appresso di molti, simili straordinari segni non essere giudicati di momento alcuno;

I6. Il reprend d'ailleurs cette double explication (colère de Dieu et erreurs des hommes) dans sa lettre de dédicace à Cosimo de' Medici : «sono stato dipoi sospeso, se io dovessi sotto il vostro felicissimo nome publicare la più mesta, la più spaventevole e la più vergognosa tragedia che la onnipotente Sapienza abbi ancora sopra questa infortunata Italia, e quasi mondana scena, dimostro; la quale, benchè in quelli infelicissimi giorni scrivessi, non per pigliare allora piacere con la penna discorrendo fra tante e tante miserabili crudeltà, ma per aver continuamente avanti agli occhi miei un manifesto esempio di quanto male sia cagione la superbia e immoderata ambizione, e quanto temere si debba, gravemente errando, la divina giustizia.» (Sacco..., p. 7-8) 
benchè le antiche istorie, e le moderne ancora, si trovino piene di queste sorti portenti, intervenuti avanti gli orrendi casi e distruzioni di città; e come per il timore di essi, quando apparivono, si legga, molti popoli cristiani, non che gentili, non aver mancato con sacrifizi e devote cerimonie placare la giusta ira del sommo Giove; la benignità del quale pare voglia istruire i mortali innanzi al flagello con diversi e spaventevoli segni, per tentare prima di ridurre con tali terrori, più tosto che con la giustizia, le umane menti a miglior vita. Nondimeno non resterò di scriverne due sopra gli altri evidentissimi, successi non molti mesi innanzi in Roma. Il primo, una saetta avere levato dal braccio di una devotissima Nostra Donna, collocata nella chiesa di Santa Maria Traspontina, il suo Bambino, e averne fatti molti pezzi, e la corona ancora di lei, in quella furia percossa e divisa in più parti, gittato per terra. Il secondo, l'eucarestia riposta il Giovedi santo preterito, come si costuma in tal giorno, in un tabernacolo della cappella del papa, la mattina seguente si trovò, senza sapere come nè da chi, sospinta per terra. Segni certamente efficacissimi da spaventare ragionevolmente ciascun cristiano, avendo il celeste fuoco tocco e guasto l'immagine della umana origine del Nostro Salvatore, e lacerato e spezzato indegnamente il glorioso premio della sua santissima Madre; e circa due mesi avanti, quello che noi cristiani meritamente tanto adoriamo, avere ricusato dimorare dove molti e molti anni prima in simil giorno stare soleva. $\mathrm{Ma}$ troppo in quella città era indurato il cuore delli scribi e farisei, per esser totalmente accecati e immersi nella voluttà, avarizia e ambizione, poichè per queste tante divine dimostrazioni non si commossero. (Sacco..., p. I78-I80; je souligne.)

On a déjà trouvé, en particulier chez Machiavel, dans les Discours (I, LVI), cette idée de l'annonce par le ciel des événements extraordinaires; Luigi Guicciardini se situe donc dans une longue tradition quand il cite les portenti, les segni evidentissimi qui ont précédé l'événement. Une fois encore, les raisons qui expliquent que rien n'ait été compris ni tenté sont les erreurs des hommes "totalmente accecati e immersi nella voluttà, avarizia e ambizione»; la dimension morale incontestable de ces erreurs des scribi e farisei est cependant à mettre en rapport avec le passage du proemio qui parle du caractère pernicieux du gouvernement des prélats (Sacco..., p. I7-I8) : «in lei [= Roma] ora solo essere concorso tanta viltà e pigrizia a ruinarla, con tanto poco pensiero di fare nel debito tempo le provisioni necessarie alla difesa sua, che facilmente ha dimostro a ciascuno, quanto l'avaro, ambizioso ed oziosissimo governo de' moderni prelati sia a' populi pernizioso.» Les vices sont les mêmes et ils ont provoqué le désastre parce que les mesures nécessaires à la défense de la ville n’ont pas été prises.

\section{«Gli atrocissimi accidenti dell'anno I527 : : le récit du sac de Rome par Francesco Guicciardini}

Francesco Guicciardini relate, dans les livres XVII et XVIII de la Storia d'Italia, la guerre menée par la Sainte Ligue (il était alors lieutenant du pape aux armées), et le sac de Rome; ces pages sont un bel exemple du 
choix de l'analyse minutieuse de l'action des hommes. Les atrocissimi accidenti $^{17}$ qui se déroulent en 1527 ne sont jamais reliés par Guicciardini à une volonté divine; ainsi, à aucun moment du texte, contrairement à d'autres passages de la Storia d'Italia (voir supra), il n'est fait mention des straordinari segni que son frère avait estimé nécessaire de rappeler; Guicciardini s'en tient à une lecture des faits, relate les débats et les décisions des chefs militaires, met en évidence les effets de ces décisions. Les analyses de Messer Francesco, soulignent les erreurs dans la fondation même de la ligue ("Dalle quali cose male intese nacque, come di sotto si dirà, principio grande di mettere in disordine la impresa che con tanta speranza si cominciava»), les cattivi consigli de Clément VII ${ }^{\mathrm{I}}$, les modi sinistri du duc d'Urbin ${ }^{19}$, à Rome même, "la debolezza grandissima dé ripari ma eziandio la mala resistenza che fu fatta dalla gente» et du côté des Impériaux et de Bourbon la détermination qui naît de la nécessité absolue ("deliberato $o$ di morire o di vincere perché certamente poca altra speranza restava alle cose sue»). Il y a là ce qu'on peut nommer une analyse laïque, sans trace de providentialisme, du processus militaire qui mène au sac de Rome. Cette nécessité d'une analyse laïque est d'ailleurs mise en évidence par Guicciardini lui-même dans un passage de sa Consolatoria où il se justifie d'avoir défendu l'hypothèse de la nécessité de mener la guerre contre l'empereur en rappelant que

se lo evento è stato diverso dal gindicio, non per questo si debbe dare colpa a chi avessi consigliato la guerra, poi che le ragione erano tale che lo persuadevano a ogni savio:

17. F. Guicciardini, Storia d'Italia, éd. de Silvana Seidel Menchi, Turin, Einaudi, I97I, XVIII, I, p. I8I7 : "Sarà l’anno mille cinquecento ventisette pieno di atrocissimi e già per piú secoli non uditi accidenti: mutazioni di stati, cattività di príncipi, sacchi spaventosissimi di città, carestia grande di vettovaglie, peste quasi per tutta Italia grandissima; pieno ogni cosa di morte di fuga e di rapine."

18. Storia d'Italia, XVIII, v, p. I838-1839: "Arrivò poi il viceré a Roma; per la venuta del quale il pontefice, giudicandosi assicurato del tutto della osservanza della concordia, licenziò con pessimo consiglio tutte le genti che nelle parti di Roma erano agli stipendi suoi, riservandosi solamente cento cavalli leggieri e dumila fanti delle bande nere"; XVIII, vI, p. I847 : "Ma era superfluo l'usare col pontefice queste diligenze: il quale, credendo troppo a quello desiderava, e troppo desiderando di alleggerirsi della spesa, subito che ebbe avviso della conclusione fatta in Firenze, con la presenza e consentimento del mandatario di Borbone, aveva imprudentissimamente licenziati quasi tutti i fanti delle bande nere; $e$ Valdemonte, come in sicurissima pace, se ne era andato per mare alla volta di Marsilia"; XVIII, vIII, p. I854: "[il papa], con cattivo consiglio, aveva licenziato prima i svizzeri e poi i fanti delle bande nere, e ricominciato si lentamente (disperato che fu l'accordo) a provedersi"; XVIII, vIII, p. I857: «lasciò indietro infelicemente il consiglio di partirsi; non stando egli e $i$ suoi capitani manco irresoluti nelle provisioni del difendersi che fussino nelle espedizioni».

19. Storia d'Italia, XVIII, IV, p. I83I: "Ma il luogotenente, comprendendo, parte da quello che era verisimile parte per relazione di parole dette da lui, che a questi modi sinistri lo induceva anche il desiderio della recuperazione del Montefeltro e di Santo Leo posseduto da fiorentini..."; XVIII, v, p. I838 : "Spaventavanlo le variazioni e il modo del procedere del duca d'Urbino". Contrairement à son frère, Francesco Guicciardini ne va pas jusqu'à accuser nettement le duc d'Urbin d'avoir voulu consciemment la défaite du pape Clément VII par haine des Médicis. Il se contente d'écrire que les décisions du duc et en particulier sa tardità (sa lenteur, à opposer à la celerità de Bourbon) sont prises o per arte o per natura et de mettre en évidence, en relatant les 
altrimenti a troppo dura condizione sarebbono sottoposti e’ consiglieri de' principi, se fussono obligati a portare in consiglio non solo discorsi e considerazione umane, ma ancora o giudicii di astrologi, o pronostici di spiriti, o profezie di frati ${ }^{20}$.

Cependant, au terme de l'analyse historique et politique restent, dans deux incises du discours historique, deux interrogations dont il faut rendre compte. La première se place au moment où Guicciardini, relatant les faiblesses des mesures prises pour défendre Rome (le piccole provisioni di Roma), dit son étonnement devant l'attitude du pape :

Ma non fu manco maraviglioso, se è maraviglia che gli uomini non sappino o non possino resistere al fato, che il pontefice, che soleva disprezzare Renzo da Ceri sopra tutti gli altri capitani, si rimettesse ora totalmente nelle sue braccia e nel suo giudizio; e molto piú che, solito a temere ne' minori pericoli, era stato piú volte inclinato ad abbandonare Roma quando il viceré andò col campo a Frusolone, ora, in tanto pericolo, spogliatosi della natura sua, si fermasse costantemente in Roma, e con tanta speranza di difendersi che, diventato quasi come procuratore degli inimici, proibisse non solo agli uomini di partirsene ma eziandio ordinasse non fussino lasciate uscirne le robe, delle quali molti mercatanti e altri cercavano per la via del fiume di alleggierirsi ${ }^{21}$.

On trouve la seconde de ces interrogations un peu plus avant dans le texte, lorsque Guicciardini esquisse le récit des calamità di Roma lors du sac; il fait une des très rares allusions aux jugements de Dieu présentes dans le récit du $\operatorname{sac}^{22}$ en relatant les viols des femmes de Rome et des religieuses par les soldats impériaux : "Sentivansi i gridi e urla miserabili delle donne romane e delle monache, condotte a torme da' soldati per saziare la loro libidine: non potendo se non dirsi essere oscuri a' mortali i giudizi di Dio, che comportasse che la castità famosa delle donne romane cadesse per forza in tanta bruttezza e miseria ${ }^{23}$."

La question des décisions divines et de leur sens éventuel est donc posée $^{24}$; elle ne l'est pas en termes providentialistes puisque, si ces événe-

débats contradictoires entre le duc et le lieutenant du pape (i.e. lui-même), les nombreux cas où le duc refuse de prendre les mesures qui auraient pu éviter l'avancée victorieuse des troupes impériales.

20. Consolatoria, ouvr. cité, p. I06-I07.

2I. Storia d'Italia, XVIII, viII, p. I855-I856.

22. Il y a une autre allusion aux avertissements divins en Storia d'Italia, XVIII, xv, p. I900 où il déclare que le pape parle comme doit le faire quelqu'un qui a "avuto da Dio sí gravi e si aspre ammonizioni».

23. Storia d'Italia, XVIII, viII, p. I859 (je souligne).

24. Que ce soit sur ce point du viol des religieuses et des femmes de Rome que se greffe l'interrogation de Guicciardini, n'est pas un hasard. Dans le Dialogo de las cosas acaecidas en Roma d'Alfonso de Valdés, écrit pour démontrer que l'empereur n'est aucunement responsable du sac de Rome et que la victoire des impériaux a été voulue par Dieu, Latancio, porte-parole de l'auteur, alors qu'il ne cesse de louer la justice de Dieu à l'œuvre lors du sac de Rome ("Verdaderamente, grandes son los juicios de Dios. Agora conozco que con el rigor de la pena recompensa la tardanza del castigo", p. 23I), a un peu de mal à expliquer que le viol des religieuses et des jeunes filles découle d'un jugement de Dieu, il s'en tire avec un "c'est la guerre qui veut ça» : 
ments sont peut-être le résultat d'un jugement de Dieu, ils restent pour le moins obscurs pour les hommes qui peuvent à bon droit se demander pourquoi Dieu a laissé advenir le mal. On peut parler d'un net refus d'une perspective providentialiste de la part de Guicciardini, refus qui passe par une séparation entre le monde de la croyance religieuse et celui de l'agir politique : les jugements de Dieu sont obscurs, ses décisions si profondes qu'il est inutile de chercher à les interpréter ${ }^{25}$; on ajoutera que dans le Dialogo del reggimento di Firenze, Francesco avait affirmé la nécessaire coupure entre le monde de l'agir politique et celui de la croyance religieuse : "chi vuole vivere totalmente secondo Dio, può mal fare di non si allontanare totalmente dal vivere del mondo, e male si può vivere secondo el mondo sanza offendere Dio".

Quant à la première interrogation, qui porte sur la possibilité ou non de "resistere al fato", elle demeure une des questions que l'ensemble des penseurs florentins auxquels nous avons fait allusion se pose, d'autant qu'elle reprend consciemment une méditation déjà présente dans l'Antiquité; on pense au premier chef à Machiavel et notamment aux Discours (II, XXIX) "La fortuna acceca gli animi degli uomini, quando la non vuole che quegli si opponghino a' disegni suoi» dans lequel Machiavel commente Tite-Live ("Adeo obcaecat animos fortuna, cum vim suam ingruentem refringi non vult»). Cette interrogation est le point d'arrivée de la réflexion de nos auteurs sur le sens à donner aux signes divins et aux catastrophes, point d'arrivée qui a deux conséquences : il y a incontestablement une limite à l'analyse rationnelle des événements et donc à l'agir politique et militaire des hommes et, en même temps, cette limite n'empêche pas un choix politique et éthique fondamental portant sur la nécessité d'agir comme si cette possibilité que la fortune ne rende vaine l'action menée n'existait pas. Ce choix nécessaire, Machiavel, précisément comme conclusion du chapitre II, XxIX des Discours, l'exprime par la formule «[gli uomini $]$

"Arcidiano: Así Dios me salve que tenéis la mayor razón del mundo. Pero si viérades aquellos soldados cómo llevaban por las calles las pobres monjas, sacadas de los monesterios, y otras doncellas, sacadas de casa de sus padres, hobiérades la mayor compasión del mundo.

Latancio: Eso es tan común cosa entre soldados y gente de guerra, que seyendo a mi parecer muy más grave que todas esas otras juntas, no hacemos ya caso dello, como si no fuese peor violar una doncella, que es templo vivo donde mora Jesucristo, que no una iglesia de piedra o madera. Pero la culpa desto no tanto se debe de echar a los soldados cuanto a vosotros, que comenzastes y levantastes la guerra y fuistes causa que ellos hiciesen lo que han hecho. Verdaderamente, aunque ningún otro mal causase la guerra, por sólo esto la debiamos de dejar." (p. I88) Les citations sont tirées d'Alfonso De Valdés, Dialogo de las cosas acaecidas en Roma, Rosa Navarro Duran (éd.), Madrid, Catedra, 1992.

25. Ricordi, C 92, p. $\mathrm{IO}_{3}$ : "Non dire: Dio ha aiutato el tale perché era buono: el tale è capitato male perché era cattivo; perché spesso si vede el contrario. Né per questo dobbiamo dire che manchi la giustizia di Dio, essendo e consigli suoi si profondi che meritamente sono detti abyssus multa." 
debbono, bene, non si abbandonare mai» dont la netteté se passe de longs commentaires. Francesco Guicciardini, quant à lui, expose sa position dans le ricordo A 136 :

Se bene gli uomini deliberano con buono consiglio, gli effetti però sono spesso contrarii; tanto è incerto el futuro. Nondimeno non si vuole come bestia darsi in preda della fortuna, ma come uomo andare con la ragione; e chi è bene savio ha da contentarsi piú di essersi mosso con buono consiglio, ancora che lo effetto sia stato malo, che se in uno consiglio cattivo avessi avuto lo effetto buono ${ }^{26}$.

Il faut, selon Messer Francesco, agir selon la raison et l'homme sage doit en quelque sorte résister à la fortune en acceptant l'échec éventuel dès lors que le consiglio qu'il a formulé est conforme à l'analyse raisonnable qu'il a effectuée. Si l'absence d'une rédaction de ce ricordo dans la dernière série écrite en 1530 peut laisser penser que l'expérience des revers subis fait douter Guicciardini, d'autres formulations ultérieures montrent qu'il s'agit d'un élément qu'il se refuse à abandonner. On pense notamment à certains passages de la Consolatoria (écrit précisément après l'échec de la guerre "pour la liberté de la pauvre Italie» et le sac de Rome) dans lesquels cette volonté d'analyser selon la raison est, de fait, une consolation face à l'échec durement subi : Guicciardini n'est pas en faute dès lors qu'il a donné un conseil raisonnable et qu'il a fait ce qui était en son pouvoir pour le réaliser dans les faits ${ }^{27}$.

Nos auteurs estiment donc que le pouvoir de la fortune et ses variations sont incontestables, elles peuvent l'emporter sur la raison et provoquer événements extraordinaires voire catastrophes; il n'en demeure pas moins que l'homme sage doit, malgré le risque de l'imprévu et de l'échec, non si abbandonare mai, andare con la ragione et refuser de darsi in preda della fortuna. Cette posture éthique, qui va de pair avec une volonté permanente d'analyse politique concrète des situations, est le seul moyen d'échapper à l'effarement et au renoncement face aux "événements extraordinaires», aux miserande tragedie et aux atrocissimi accidenti.

26. Ricordi, A I36; B I60, p. 252.

27. Consolatoria, ouvr. cité, p. I07: "Non sei adunche in colpa se al consiglio che tu avessi dato della guerra non ha corrisposto lo evento; anzi meriti laude e non piccola, perché come sa chi è stato vicino alle azioni tue, hai quanto ti è stato possibile aiutato che lo effetto non sia stato diverso dalla ragione; e tanto che se gli altri che hanno avuto carico nella guerra avessino fatto nel grado loro quanto hai fatto tu nel tuo, o se el papa poi che era entrato nel mare avessi nel navigare seguitato e' ricordi tuoi, forse che le cose arebbono avuto altro fine di quello che hanno avuto. Non ci è adunche colpa tua né nel consiglio poi che l'hai dato ragionevole, né nello evento poi che di quello che era in potestà tua non gli sei mancato; e però ritrovandoti da ogni parte innocente e sanza errore, ti debbi anche ragionevolmente trovare sanza dispiacere." 


\section{Bibliographie}

Abulafia David (éd.), The French Descent into Renaissance Italy I494-95: Antecedents and Effects, Aldershot (G.B.), Variorum, I995.

Albertini Rudolf von, Firenze dalla repubblica al principato, Turin, Einaudi, 1995 [ I ${ }^{\text {re }}$ éd. Bern, I955].

Chastel André, Le sac de Rome, I527, Paris, Gallimard, 1984.

Commynes Philippe de, Mémoires, édition critique par Joël Blanchard, Genève, Droz, 2007.

De Valdés Alfonso, Dialogo de las cosas acaecidas en Roma, Rosa Navarro Duran (éd.), Madrid, Catedra, 1992.

Guicciardini Francesco, Ricordi, edizione critica a cura di Raffaele Spongano, Florence, Sansoni, 1951.

—, Consolatoria, dans Autodifesa di un politico, Bari-Rome, Laterza, 1993.

—, Storia d'Italia, éd. de Silvana Seidel Menchi, Turin, Einaudi, I97I.

Guicciardini Luigi, Il sacco di Roma, dans Carlo Milanesi (éd), Il sacco di Roma del MDXXVII. Narrazioni di contemporanei scelte per cura di Carlo Milanesi, Florence, G. Barbèra Editore, I867.

Machiavelli Niccolò, Opere, éd. de C. Vivanti, Turin, Einaudi, 1999.

Moreno Paola, Godinas Laurette et Miesse Hélène (éd.), Encuentros / Desencuentros. Italia y España en los siglos XV y XVI: Textos y contextos. Actas del congreso internacional (UNAM-Instituto de Investigaciones bibliogràficas, 26-27 octobre 2009), sous presse. 